\title{
Usefulness of Four-Dimensional Trans-Oesophageal Echocardiography in Device Closure of An Unusual Post Myocardial Infarction Ventricular Septal Defect
}

\author{
Gregory James Skinner ${ }^{1}$, Christopher Duke ${ }^{1,2}$, and Suhair Omar Shebani ${ }^{1 *}$ \\ ${ }^{1}$ East Midlands congenital heart centre, Glenfield Hospital, Leicester, LE3 9QP, UK \\ ${ }^{2}$ Department of Congenital Heart Disease, Sheikh Khalifa Medical City, Abu Dhabi, UAE
}

Corresponding author: Suhair O Shebani, East Midlands congenital heart centre, Glenfield Hospital, Leicester, LE3 9QP, UK, Tel: + 0044300303 1573; E-mail: suhair.shebani@uhl-tr.nhs.uk

Rec Date: Feb 06, 2017, Acc Date: Feb 12, 2017, Pub Date: Feb 14, 2017

Citation: Skinner JG, Duke C, Shebani SO. Usefulness of Four-Dimensional Trans-Oesophageal Echocardiography in Device Closure of An Unusual Post Myocardial Infarction Ventricular Septal Defect. Interv Cardiol J 2017, 3:1.

\section{Abstract}

Presentation of the transoesophageal echocardiogram for a patient undergoing transcatheter closure of a post infarction ventricular septal defect through a pseudoaneurysm at the basal rather than the apical part of the septum, posing a diagnostic and interventional planning challenge. Four dimensional imaging clarified the complex anatomy of the pseudoaneurysm, aiding device closure.

Keywords: 4D echocardiography; Transoesophageal echocardiography; Post infarction ventricular septal defect; Device closure of ventricular septal defect

\section{Introduction}

An 80-year-old gentleman was admitted with an inferior myocardial infarction complicated by a ventricular septal defect (VSD), resulting in acute severe congestive heart failure. Coronary angiography demonstrated occlusion of the right coronary artery at the crux (Video Supplement 1). A transthoracic echocardiogram (TTE) and a contrast computed tomography (CT) scan demonstrated the position of the defect in the basal inferior septum, although did not clearly delineate the precise anatomy of the defect (Figure 1). He was discussed at a multidisciplinary meeting, where he was deemed too high risk for surgical closure, and was put forward for transcatheter closure as a salvage procedure. Informed consent was given, with the high mortality risk understood by the patient and his family.

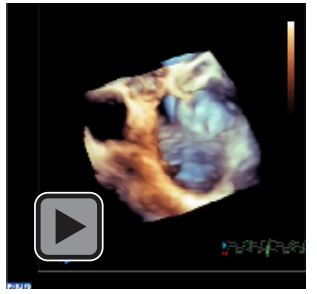

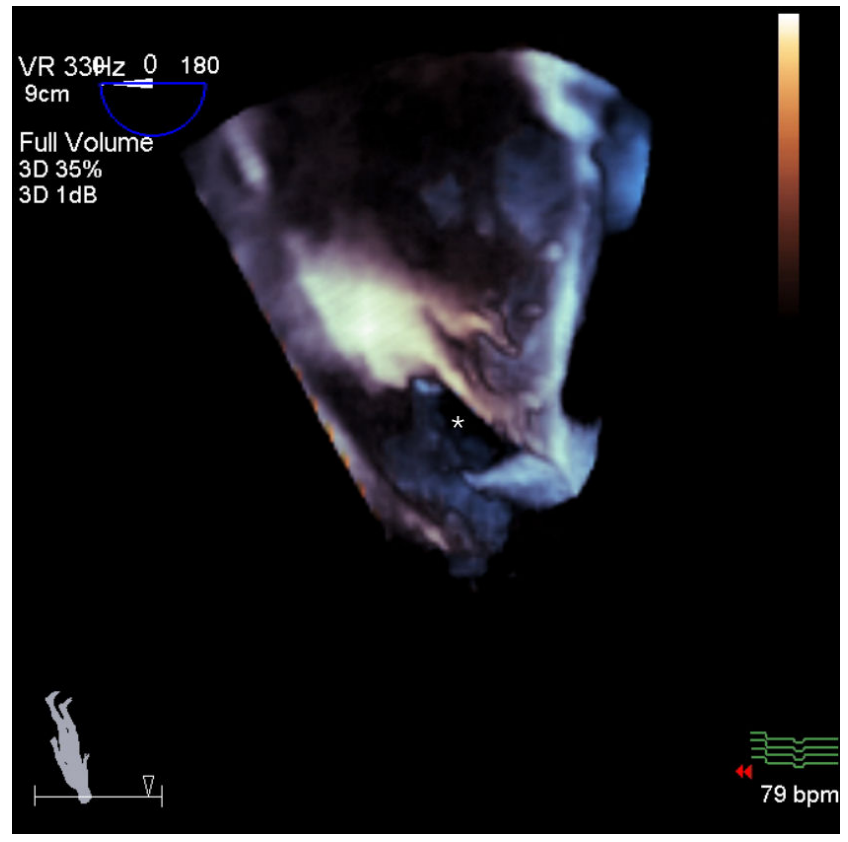

Figure 1 Axial contrast CT scan demonstrating the VSD $\left({ }^{*}\right)$ and the pseudoaneurysm $(\Delta)$. Note the difficulty clearly delineating the precise anatomy of the defect.

He underwent a transcatheter device closure of the VSD with four-dimensional (4D) trans-oesophageal echocardiogram (TOE) guidance (Figures 2 - 4).

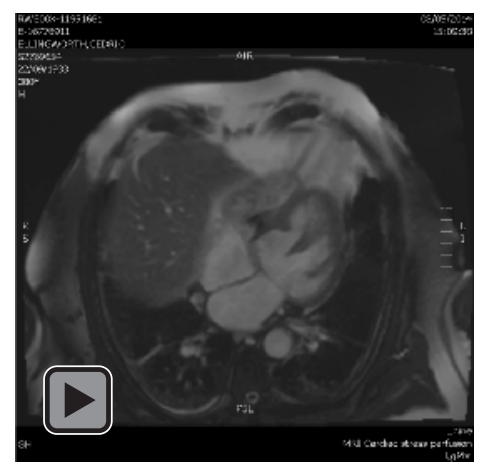




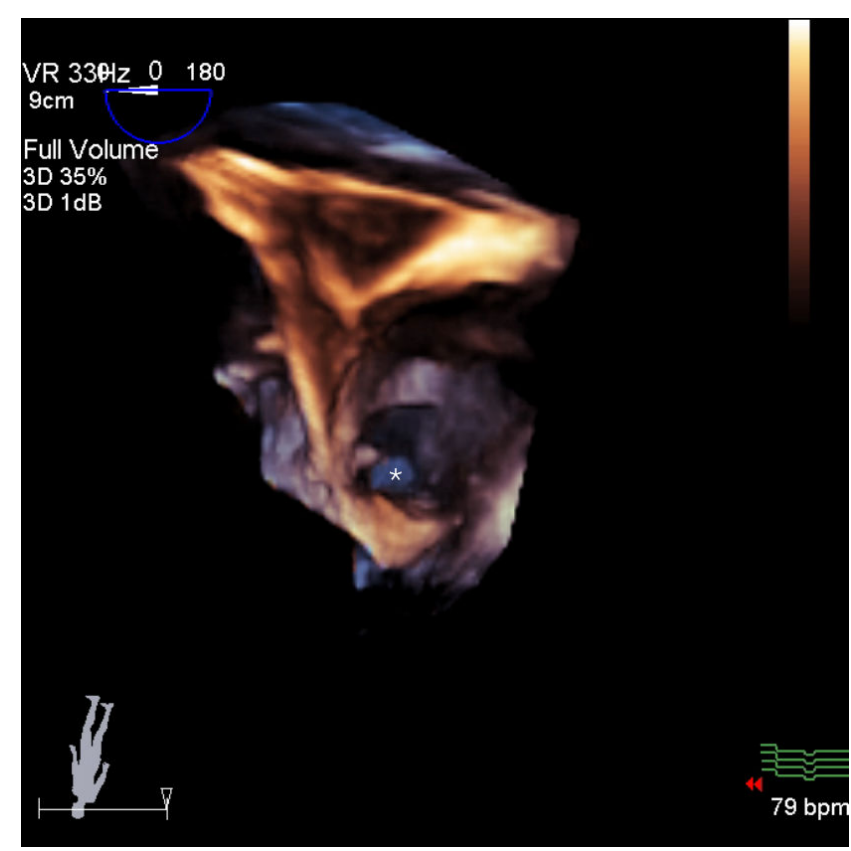

Figure 2 3D view from the right ventricular aspect with the inferior wall of the right ventricle peeled off by the pseudoaneurysm (* - VSD).

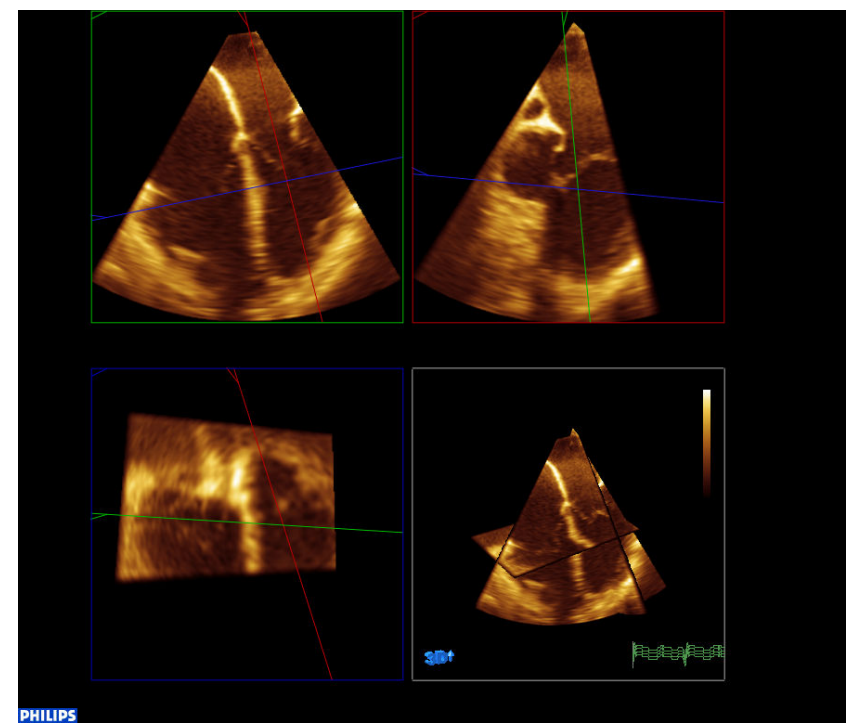

Figure 3 Pseudoaneurysm from the left ventricular aspect (* - VSD).

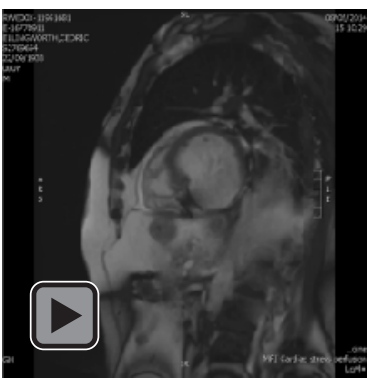

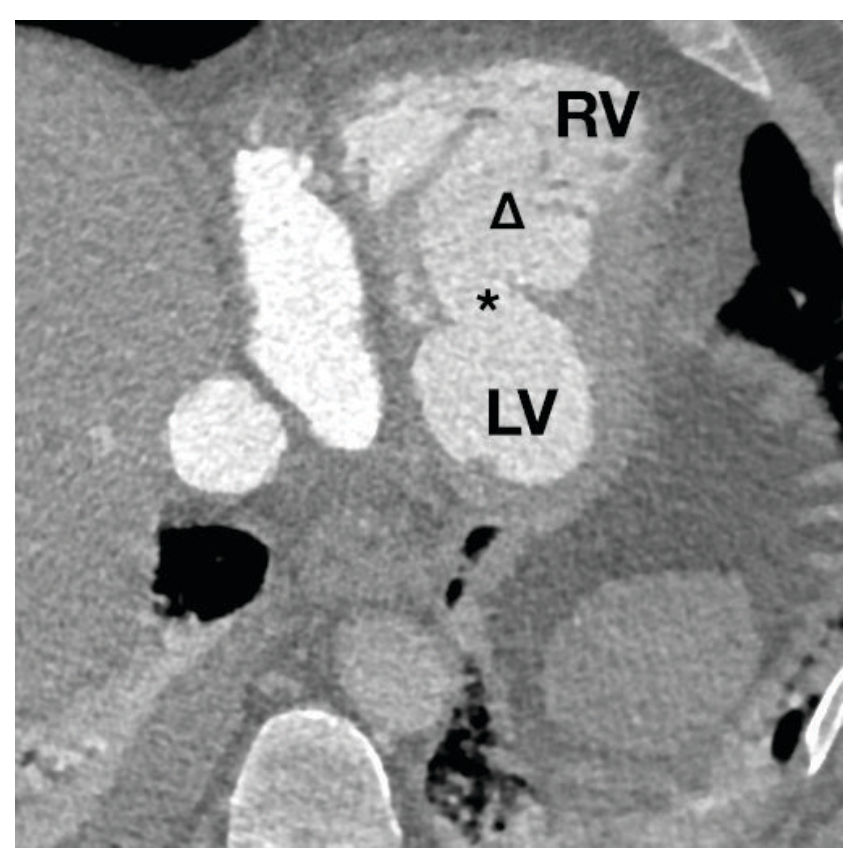

Figure 4 Multi-plane reconstruction of the aneurysm with device in situ, isolated from the tricuspid valve by peeled-off tissue.

The TOE demonstrated the VSD in the inferoseptal area. The defect was complex, as a pseudoaneurysm had formed from the left ventricular basal inferior septum into the inferior wall of the right ventricle. This pseudoaneurysm then opened into the right ventricle more apically, leaving a flap of the right ventricular inferior wall between the communicating passage and the tricuspid valve. The interventional cardiologists were able to deploy a device (a $24 \mathrm{~mm}$ Amplatzer muscular VSD occluder) within this aneurysmal passage with no danger of it impinging on the tricuspid valve (Video Supplement 2).

Rapid improvement followed, and at 6 weeks follow up the patient was once again independent and riding his bicycle. He remains well and active on follow up, nearly three years after the procedure. A follow up magnetic resonance imaging (MRI) scan demonstrated the device in position within the pseudoaneurysm and significant improvement in cardiac function (Video Supplement 3).

Due to the complex three-dimensional nature of this defect, it was extremely difficult to conceptualise the anatomy with only the standard B mode images nor on angiography. Using 4D TOE volume rendering and multi-plane reformatting (MPR) live during the case allowed the anatomy of the defect to be appreciated by both the imaging and interventional teams, and for guidance for the device closure, which was very successful in a safe position with no residual shunt. This case clearly shows the added value of 4D imaging with challenging structural heart disease. 